\title{
Should transanal total mesorectal excision be implemented in medium-sized colorectal unit? technical and oncological outcome
}

\author{
Man-fung $\mathrm{Ho}^{1,2}$, Dennis Chung-Kei $\mathrm{Ng}^{2}$, Janet Fung-yee Lee ${ }^{1}$, Simon Siu-man $\mathrm{Ng}^{1}$ \\ ${ }^{1}$ Department of Surgery, Prince of Wales Hospital, The Chinese University of Hong Kong, Hong Kong Special Administrative Region; \\ ${ }^{2}$ Department of Surgery, North District Hospital, Hong Kong Special Administrative Region, China
}

Purpose: This study was performed to evaluate the outcome of implementation of transanal total mesorectal excision (TaTME) for low rectal cancer in a regional hospital and in comparison to laparoscopic (Lap) TME.

Methods: Consecutive patients with low rectal cancer of which the lowest border of the tumour was located beween 1 and 5 $\mathrm{cm}$ from the puborectalis who underwent TME at North District Hospital between January 2013 and December 2019 were included. Clinical, operative, and pathologic outcomes were compared between Lap TME and TaTME. The primary end point was complication profile.

Results: Thirty-five patients underwent Lap TME and 45 patients underwent TaTME for low rectal cancer. The conversion rate of the TaTME group was significantly lower than that of the Lap TME group ( $4.4 \%$ vs. $20 \%, \mathrm{P}=0.029)$, but the operating time was longer (259 minutes vs. 219 minutes, $\mathrm{P}=0.009$ ). The tumour location was significantly lower in the TaTME group, but the distal resection margins were adequate and not different between both groups. The TaTME group had higher incidence rates of prolonged ileus and urinary tract infection, but the other complications were similar between the two groups. The resection margin positivity rates of the TaTME and Lap TME groups were $2.2 \%$ and $5.7 \%$, respectively $(\mathrm{P}=0.670)$. At a median follow up of 39 months, no abnormal early recurrence was detected.

Conclusion: It is technically feasible and oncologically safe to perform TaTME in a medium-volume colorectal unit. Patients with difficult pelvic anatomy can benefit by reducing the risk of conversion and margin positivity rate.

Keywords: Rectal neoplasms; Laparoscopy; Adenocarcinoma; Digestive system surgical procedures; Treatment outcome

\section{INTRODUCTION}

Total mesorectal excision (TME) is the gold standard for treatment of rectal cancer, and it can be performed using open, laparoscopic, or robotic approaches. Transanal TME (TaTME) as a new

Received: Nov 4, 2020 - Revised: Jan 25, 2021 - Accepted: Feb 23, 2021 Correspondence to: Man-fung Ho, MBChB (CUHK), FRCSEd (Gen), FCSHK, FHKAM (Surgery)

Department of Surgery, Prince of Wales Hospital, 4/F, Clinical Sciences Building, Shatin, Hong Kong Special Administrative Region, China Tel: +852-97478731

E-mail:mfho@surgery.cuhk.edu.hk

ORCID: https://orcid.org/0000-0001-6929-9723

(C) 2022 The Korean Society of Coloproctology

This is an open-access article distributed under the terms of the Creative Commons Attribution NonCommercial License (https://creativecommons.org/licenses/by-nc/4.0) which permits unrestricted noncommercial use, distribution, and reproduction in any medium, provided the original work is properly cited. surgical approach for rectal cancer has gained a lot of interest among colorectal surgeons worldwide over the last decade. Since the first clinical report of TaTME by Sylla et al. [1] in 2010, there has been an exponential growth in practice of this new approach for treatment of low rectal cancer in different parts of the world. TaTME offers surgeons the ability to overcome the difficult anatomy in the deep pelvis and the potential to achieve a better quality resection $[2,3]$. On the other hand, TaTME has also been reported to be associated with adverse outcomes including novel complications of urethral injury, injury to the lateral pelvic compartment, and atypical and early recurrence, etc. $[4,5]$. The true benefits of this novel approach for rectal cancer and its relative efficacy, when compared with other conventional approaches, have yet to be proven by ongoing randomized controlled trials such as COLOR (COlorectal cancer Laparoscopic or Open Resection) III trial and ETAP-GRECCAR 11 trial $[6,7]$. 
The evolution of TaTME started almost 40 years ago and is a combination of several important developments in minimally invasive surgery, endoscopic surgery, and transanal surgery. It began with a technique called transanal abdominal transanal (TATA) radical proctosigmoidectomy with coloanal anastomosis that was first developed in 1984 in the cadaveric laboratory at Thomas Jefferson University by Dr. Gerald Marks [8]. The main advantage of TATA is that it allows surgeons to clearly visualize and secure the distal resection margin especially when dealing with small tumors or scars after neoadjuvant chemoradiotherapy. Later, transanal endoscopic microsurgery (TEM) and transanal endoscopic operation (TEO) devices were employed for performing rectotomy in transanal and rectal procedures [7]. These endoscopic platforms provide surgeons with better visualization and magnification during rectotomy. However, both TEM and TEO are rigid platforms and can pose ergonomic difficulty to surgeons during dissection. This was later improved when the transanal minimal invasive surgery (TAMIS) flexible platforms come into play. Besides being a flexible platform, TAMIS also allows surgeons to use conventional laparoscopic instruments for rectal dissection [8].

The conventional laparoscopic TME (Lap TME) approach has technical limitations especially in patients with narrow pelvis, visceral obesity, and bulky tumor. Maintaining good visibility, performing bowel traction and dissection, and transecting the lower rectum by a currently available stapling device in the deep pelvis can often be difficult during Lap TME [5]. These technical challenges can be even more significant when dealing with low or bulky rectal tumors. TaTME has been adopted to overcome these technical challenges, and to facilitate difficult pelvic dissection, mostly in high volume centers with more resources $[9,10]$.

Our colorectal unit is a medium volume unit that performed about 30 rectal cancer resections per year. Similar to other high volume centers, we would also encounter the same difficulties during Lap TME for low rectal cancer. These technical difficulties can result in a higher complication rate, higher risk of conversion, prolonged operating time, and potentially poor surgical outcomes. To tackle these technical difficulties, our unit has introduced TaTME as the preferred surgical approach for low rectal cancer since 2016 after undertaken adequate training in 2015.

This study aims to evaluate the feasibility and safety of implementation of TaTME for low rectal cancer in a medium volume unit, and to compare the results with Lap TME.

\section{METHODS}

The study was conducted at the colorectal unit of North District Hospital in Hong Kong Special Administrative Region. Our TaTME program commenced in 2016. All patients with operable low rectal adenocarcinoma of which the lowest border of the tumor was located between 1 and $5 \mathrm{~cm}$ from the puborectalis on preoperative magnetic resonance imaging (MRI) were subjected to sphincter preserving TaTME at our unit after 2016. If the low- est border of the tumor was less than $1 \mathrm{~cm}$ from the puborectalis noted on preoperative MRI or intraoperatively, abdominoperineal resection was performed. All patients were evaluated at our multidisciplinary team meetings to determine resectability and the need for neoadjuvant therapy. Patients with threatened circumferential margin, positive extramural venous invasion, clinical N2 stage or above were subjected to neoadjuvant long course chemoradiotherapy. If posttreatment imaging confirmed resectability, surgery would be offered. Patients with resectable solid organ metastasis were also considered as having a resectable disease.

Every TaTME procedure was performed by 2 teams (abdominal and transanal) of experienced surgeons working in phases. The abdominal team consisted of a chief surgeon and an assistant, who started the operation with standard port placement (Fig. 1), followed by laparoscopic mobilization of the left colon using the medial approach, and then division of the inferior mesenteric vessels at the origin. Splenic flexure was taken down routinely to allow tension-free anastomosis. The transanal team would start the procedure only after splenic flexure mobilization had been completed by the abdominal team. The transanal team consisted of a trained TaTME surgeon and an assistant holding the camera (a lens holder would be used if assistant manpower was not available). The transanal procedure began with the placement of a Lone Star retractor (Lone Star Retractor System, CooperSurgical Inc., Trumbull, CT, USA) and the insertion of a GelPOINT path transanal access platform (Applied Medical, Rancho Santa Margarita, CA, USA) into the anal canal. This was followed by the application of a purse-string suture below the lowest border of the tumor with at least $1-\mathrm{cm}$ macroscopic distal margin. Circumferential rectotomy was then performed and the TME plane was entered. This transanal mesorectal dissection was continued in the cephalad direction and eventually joined with the abdominal team dissection at the peritoneal reflection level. The specimen was extracted from a Pfannenstiel incision. Anastomosis was performed by either a circular stapler or hand-sewn coloanal anasto-

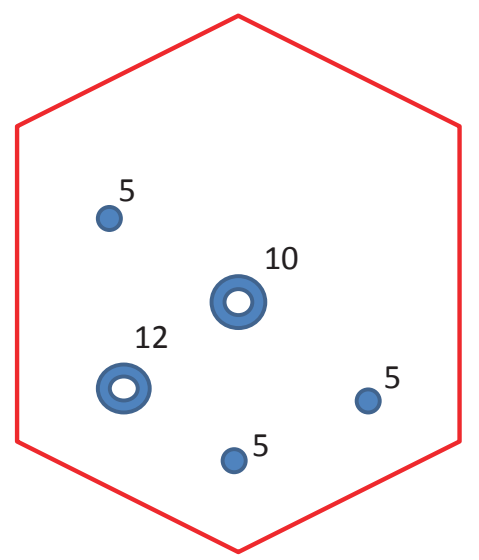

Fig. 1. Standard port placement for laparoscopic total mesorectal excision. 
mosis (Figs. 2, 3).

In this study, patients who underwent TaTME were compared with a historic cohort of patients with low rectal cancer who underwent conventional Lap TME at our unit between 2013 and 2015. The inclusion criteria were the same for both groups; those with low rectal cancer of which the lowest border of the tumor was located between 1 and $5 \mathrm{~cm}$ from the puborectalis on preoperative MRI, and those who underwent sphincter preserving TME. Patients who underwent multivisceral resection in addition to the TME surgery were excluded. The clinical, operative, pathologic, and oncologic outcomes were compared between the 2 groups.

The primary endpoint of the study was postoperative complications; and secondary endpoints included pathology of the resected specimens, recurrence rate, and survival rate.

Regarding statistical analysis, continuous variables between the 2 groups were compared by Student t-test. Categorical variables were compared using the chi-square test or Fisher exact test. Statistical significance was assumed when P-value was $<0.05$. Recurrence and survival rates were determined by Kaplan-Meier curved analysis and compared using a log-rank test.

Ethics approval granted from the Joint Chinese University of Hong Kong-New Territories East Cluster Clinical Research Ethics Committee (CREC Ref. No. 2020.180) in accordance with its

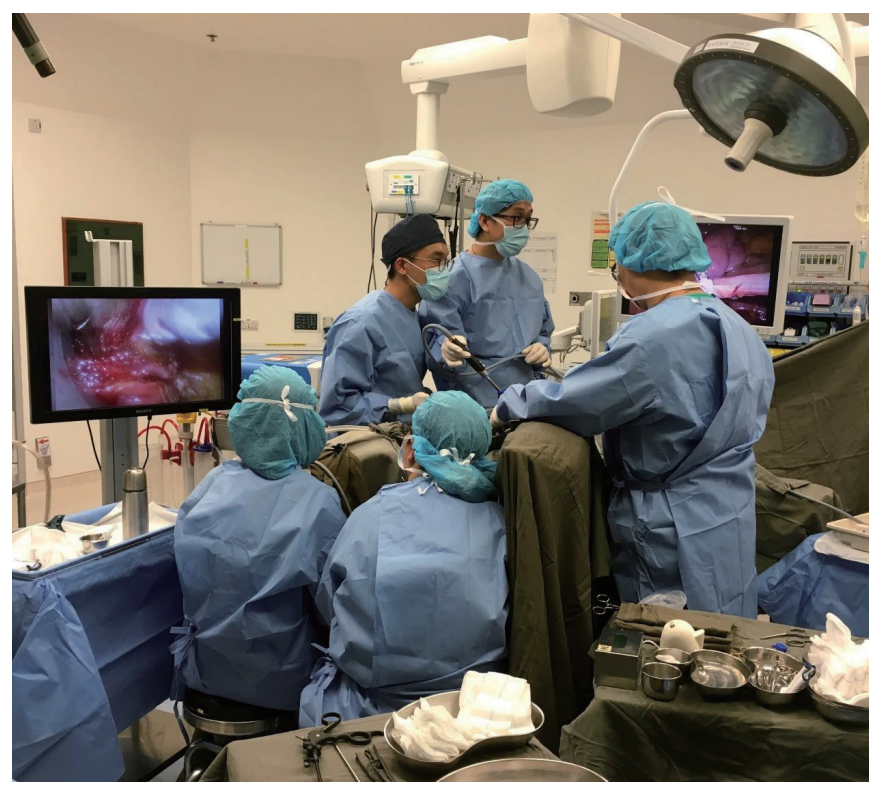

Fig. 2. Operative settings. The abdominal team over the right side of the patient as usual laparoscopic rectal surgery. Transanal team standing in between legs. In case of a shortage of manpower, transanal team surgeon can operate with a lens holder without an assistant.
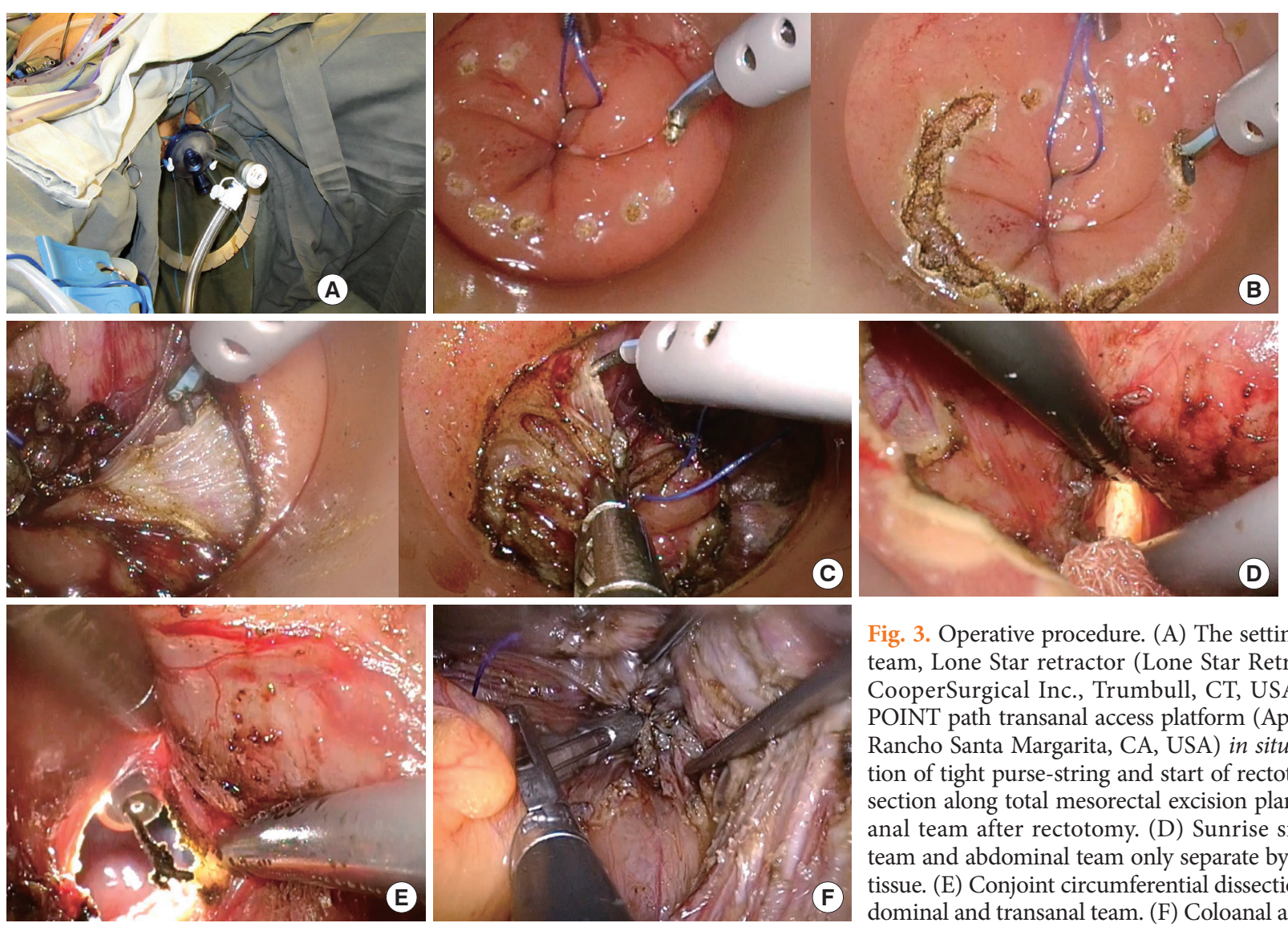

Fig. 3. Operative procedure. (A) The setting of transanal team, Lone Star retractor (Lone Star Retractor System, CooperSurgical Inc., Trumbull, CT, USA) and a GelPOINT path transanal access platform (Applied Medical, Rancho Santa Margarita, CA, USA) in situ. (B) Completion of tight purse-string and start of rectotomy. (C) Dissection along total mesorectal excision plane from transanal team after rectotomy. (D) Sunrise sign, transanal team and abdominal team only separate by 1 layer of soft tissue. (E) Conjoint circumferential dissection by both abdominal and transanal team. (F) Coloanal anastomosis. 
standard operating procedure and the principles of the Declaration of Helsinki and Good Clinical Practice of International Council of Harmonization. The written informed consent was waived for reviewing anonymous patients.

\section{RESULTS}

Between January 2013 and December 2015, 70 patients with mid and low rectal cancer underwent Lap TME at our unit. Among them, 35 patients with low rectal cancer who underwent sphincter preserving Lap TME and without multivisceral resections were included in this study as the control group for comparison.

Table 1. Demographic characteristics of patients with Lap TME vs. TaTME

\begin{tabular}{lccc}
\hline Characteristic & $\begin{array}{c}\text { Lap TME } \\
(\mathrm{n}=35)\end{array}$ & $\begin{array}{c}\text { TaTME } \\
(\mathrm{n}=45)\end{array}$ & P-value \\
\hline Sex & $28(80.0)$ & $37(82.2)$ & 0.801 \\
$\quad$ Male & $7(20.0)$ & $8(17.8)$ & \\
$\quad$ Female & $65.63 \pm 11.24$ & $65.82 \pm 9.94$ & 0.935 \\
Age (yr) & $12.22 \pm 1.79$ & $12.44 \pm 2.09$ & 0.614 \\
Initial hemoglobin (g/dL) & $12.98 \pm 23.75$ & $8.14 \pm 12.40$ & 0.243 \\
Carcinoembryonic antigen & $16(45.7)$ & $21(46.7)$ & 0.932 \\
Neoadjuvant therapy & & & 0.267 \\
ASA PS classification & $8(22.9)$ & $18(40.0)$ & \\
$\quad$ I & $22(62.9)$ & $22(48.9)$ & \\
II & $5(14.3)$ & $5(11.1)$ & \\
$\quad$ II & $22.56 \pm 3.64$ & $22.70 \pm 2.90$ & 0.855 \\
Body mass index $\left(\mathrm{kg} / \mathrm{m}^{2}\right)$ & $31(88.6)$ & $44(97.8)$ & 0.091 \\
Surgery of curative intent & $10(28.6)$ & $7(15.6)$ & 0.158 \\
\hline Permanent stoma &
\end{tabular}

Values are presented as number (\%) or mean \pm standard deviation.

Lap TME, laparoscopic total mesorectal excision; TaTME, transanal total mesorectal excision; ASA, American Society of Anesthesiologists; PS, physical status.
TaTME was adopted as the procedure of choice for the treatment of low rectal cancer at our unit since January 2016. As of December 2019, 45 patients with low rectal cancer had undergone TaTME at our unit, and these patients constituted the experimental group in this study (with the same inclusion criteria as the control group) (Fig. 4). The demographic data and baseline characteristics of the 2 groups were comparable (Table 1). The median duration of follow-up was 39 months.

Table 2 summarizes the operative characteristics and results of the 2 groups. The conversion rate of the TaTME group was significantly lower than that of the Lap TME group (4.4\% vs. $20.0 \%$, $\mathrm{P}=0.029$ ). There were 7 conversions to open surgery in the Lap TME group; 5 due to difficult pelvic conditions (including bleeding in the pelvis, visceral obesity, and difficulty in rectal transection), and 2 due to intraabdominal adhesions. In the TaTME group, 2 patients required conversion to open surgery because of intraabdominal adhesions. The TaTME group had a longer operating time when compared with the Lap TME group (258.7 minutes vs. 218.9 minutes, $\mathrm{P}=0.009$ ). Fig. 5 shows the operating time of the procedures plotted against the number of cases. The mean

Table 2. Operative characteristics of patients with Lap TME vs. TaTME

\begin{tabular}{lccc}
\hline Characteristic & $\begin{array}{c}\text { Lap TME } \\
(\mathrm{n}=35)\end{array}$ & $\begin{array}{c}\text { TaTME } \\
(\mathrm{n}=45)\end{array}$ & P-value \\
\hline Conversion & $7(20.0)$ & $2(4.4)$ & $0.029^{*}$ \\
Blood lost (mL) & $278.6 \pm 318.42$ & $252.89 \pm 148.48$ & 0.633 \\
Distal margin (cm) & $2.06 \pm 1.31$ & $1.62 \pm 0.94$ & 0.080 \\
Operative time (min) & $218.9 \pm 56.89$ & $258.7 \pm 71.35$ & $0.009^{*}$ \\
$\begin{array}{l}\text { Tumor location from external } \\
\text { sphincter (cm) }\end{array}$ & $3.56 \pm 1.10$ & $2.73 \pm 1.06$ & $0.001^{*}$ \\
Linear stapler & $3.25 \pm 1.69$ & NA & \\
\hline
\end{tabular}

Values are presented as number (\%) or mean \pm standard deviation.

Lap TME, laparoscopic total mesorectal excision; TaTME, transanal total mesorectal excision; NA, Not applicable.

${ }^{*}$ Statistical significance, $\mathrm{P}<0.05$.

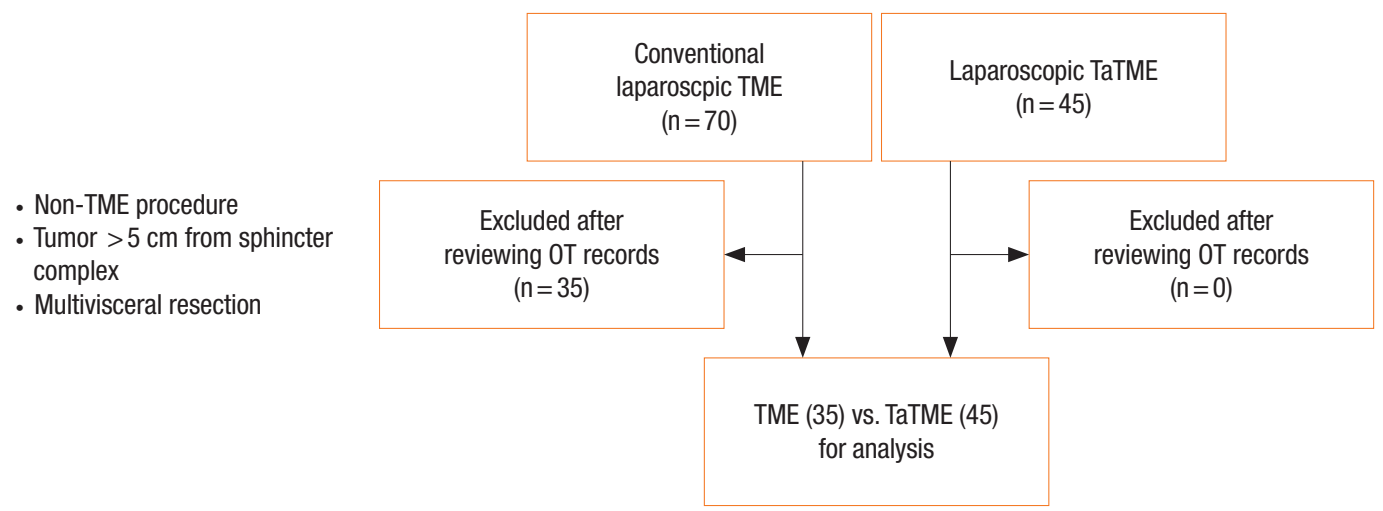

Fig. 4. Patients included and excluded for analysis. TME, total mesorectal excision; TaTME, transanal total mesorectal excision; OT, operative theatre. 
tumor location measured from the top of the puborectalis was significantly lower in TaTME group $(2.73 \mathrm{~cm}$ vs. $3.56 \mathrm{~cm}$, $\mathrm{P}=0.001)$. The mean distal margin and blood lost were similar between both groups. All except one patients had a tumor margin of at least $1 \mathrm{~cm}$ measured from the top of puborectalis on preoperative MRI. The patient with the initial suboptimal distal margin underwent neoadjuvant chemoradiotherapy and resulted in a very small residual tumor. Intraoperative assessment subsequently confirmed that the distal margin was adequate and hence TaTME was performed. The mean number of stapler firings for rectal transection in the Lap TME group was 3.25 \pm 1.69 .

Regarding pathologic characteristics, there was no significant difference in tumor $\mathrm{T}$ staging $(\mathrm{P}=0.611)$, and $\mathrm{N}$ staging $(\mathrm{P}=$ 0.401 ) between the 2 groups. The number of total lymph nodes harvested (Lap TME vs. TaTME: 11.9 vs. 13.4, $\mathrm{P}=0.345$ ) and number of positive lymph nodes (Lap TME vs. TaTME: 1.51 vs. $0.71, \mathrm{P}=0.159$ ) in both groups were also similar. Two patients in the Lap TME group (5.7\%) and 1 patient in the TaTME group $(2.2 \%)$ had positive resection margins $(\mathrm{P}=0.670)$. In the Lap TME group, 1 patient had positive distal resection margin, and 1 patient had positive circumferential resection margin. In the TaTME group, there was 1 patient with positive circumferential resection margin (Table 3 ).

The postoperative complications profile was similar between the 2 groups except for a higher incidence of postoperative ileus $(\mathrm{P}=$ $0.025)$ and urinary tract infection/acute retention of urine (UTI/ AROU) $(\mathrm{P}=0.042)$ in the TaTME group. There was no significant difference in postoperative hemorrhage, collection, anastomotic leak, wound complications, pneumonia, and reoperation rates between the 2 groups. There was no urethral or vaginal injury among patients who underwent TaTME. There was no 30-day mortality in this study. The mean hospital stay was also similar between the 2 groups (Lap TME vs. TaTME: 10.17 vs. 10.49 days, $\mathrm{P}=0.863$ ) (Table 4).
Regarding oncologic outcomes, the Kaplan-Meier plots did not show significant differences in overall survival and disease-free survival between the 2 groups (Figs. 6, 7).

\section{DISCUSSION}

Our study demonstrated that the implementation of TaTME in a medium volume unit is technically feasible and oncologically safe. TaTME is not associated with increased complications and abnormal pattern of recurrence compared with conventional Lap TME.

Table 3. Pathological characteristics of patients with Lap TME vs. TaTME

\begin{tabular}{lccc}
\hline Characteristic & $\begin{array}{c}\text { Lap TME } \\
(\mathrm{n}=35)\end{array}$ & $\begin{array}{c}\text { TaTME } \\
(\mathrm{n}=45)\end{array}$ & P-value \\
\hline T staging & $3(8.6)$ & $5(11.1)$ & 0.611 \\
0 & $0(0.0)$ & $2(4.4)$ & \\
is & $4(11.4)$ & $6(13.3)$ & \\
1 & $6(17.1)$ & $10(22.2)$ & \\
2 & $22(62.9)$ & $22(48.9)$ & \\
3 & $2(5.7)$ & $1(2.2)$ & 0.670 \\
Margin involved & & & 0.401 \\
N staging & $19(54.3)$ & $31(68.9)$ & \\
0 & $13(37.1)$ & $11(24.4)$ & \\
1 & $3(8.6)$ & $3(6.7)$ & \\
2 & $1.51 \pm 3.45$ & $0.71 \pm 1.36$ & 0.159 \\
Positive lymph node & $11.9 \pm 6.33$ & $13.4 \pm 6.79$ & 0.345 \\
\hline Total lymph node harvested & & & \\
\hline
\end{tabular}

Values are presented as number (\%) or mean \pm standard deviation.

Lap TME, laparoscopic total mesorectal excision; TaTME, transanal total mesorectal excision.

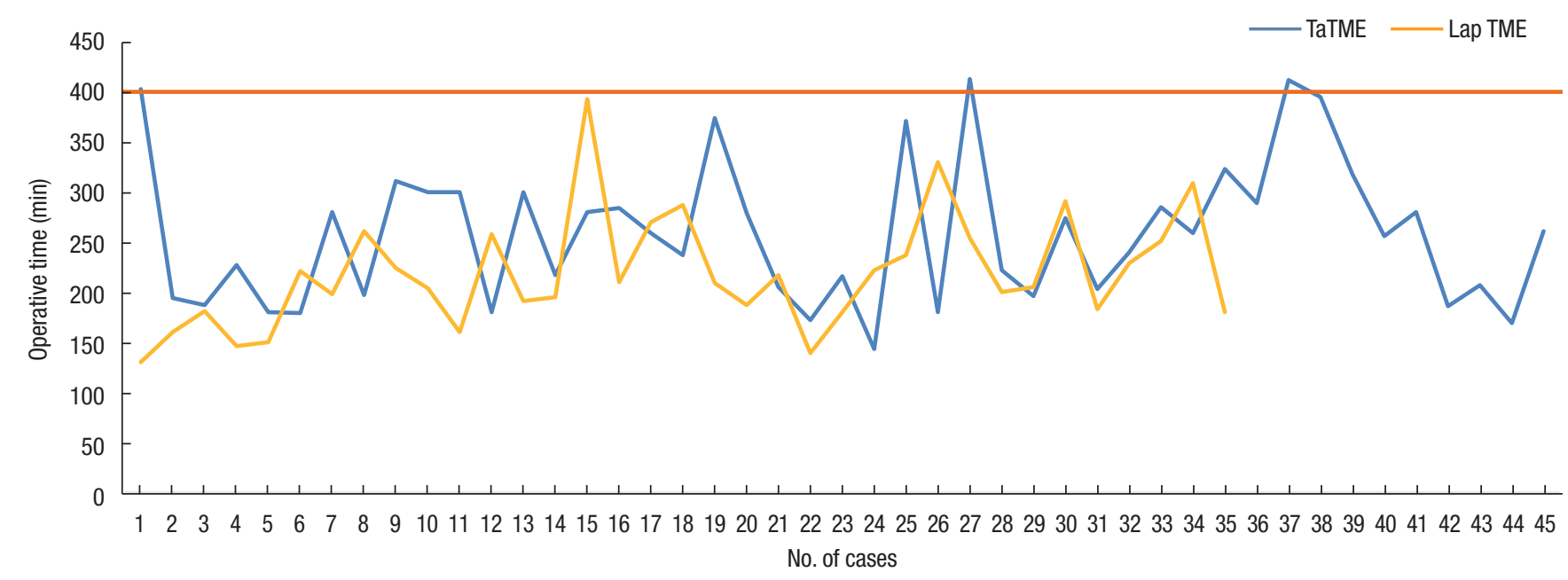

Fig. 5. Operative time against number of cases for conventional laparoscopic (Lap) total mesorectal excision (TME) and transanal TME (TaTME). 
Table 4. Postoperative complications profile of patients with Lap TME vs. TaTME

\begin{tabular}{lllc}
\hline Complication & $\begin{array}{c}\text { Lap TME } \\
(\mathrm{n}=35)\end{array}$ & $\begin{array}{l}\text { TaTME } \\
(\mathrm{n}=45)\end{array}$ & P-value \\
\hline Fever & $9(25.7)$ & $2(4.4)$ & 0.066 \\
Anastomotic bleeding & $0(0)$ & $0(0)$ & $\mathrm{NA}$ \\
$\begin{array}{l}\text { Operative site collection } \\
\text { Anastomotic leak }\end{array}$ & $2(5.7)$ & $4(8.8)$ & 0.593 \\
$\begin{array}{l}\text { Wound infection/dehiscence } \\
\text { Pneumonia }\end{array}$ & $3(8.6)$ & $3(6.7)$ & 0.748 \\
$\begin{array}{l}\text { Ileus } \\
\begin{array}{l}\text { Urinary tract infection/retention } \\
\text { of urine }\end{array}\end{array}$ & $0(0)$ & $0(0)$ & $\mathrm{NA}$ \\
Reoperation & $0(0)$ & $6(13.3)$ & $0.025^{\star}$ \\
\hline
\end{tabular}

Values are presented as number (\%).

Lap TME, laparoscopic total mesorectal excision; TaTME, transanal total mesorectal excision; NA, not applicable.

*Statistical significance, $P<0.05$

The new approach can benefit those patients with difficult pelvic anatomy by reducing the risk of conversion and potentially decreasing the resection margin positivity rate in particular the distal resection margin. It also helps surgeons tackle difficult rectal transection and increases the chance of sphincter preservation for low rectal cancer.

We are a medium volume colorectal unit with an average of 180 colorectal cancer operations per year, including 30 rectal cancer resections. Various cutoffs to define a high volume center for rectal cancer surgery have been used in the literature and these range from 17 to 35 cases per year [11]. According to our local data, a high-volume center usually performs 60 TMEs per year while a medium volume center performs 20 TMEs per year [12]. In a systematic review evaluating the clinical outcomes of TaTME according to case volume effect, the conversion rate, circumferential margin positivity rate, and major complication rate in low volume centers (those that performed less than 30 cases per year) were $4.3 \%, 4.8 \%$, and $12.2 \%$, respectively [13]. Our center's results are probably more promising than those low volume centers in the systematic review and comparable to those high volume centers in terms of conversion rate, complication rate, and specimen quality.

Since the introduction of TaTME in our unit in 2016, we have been selecting those patients with low rectal cancer of which the lowest border of the tumor is located between 1 and $5 \mathrm{~cm}$ from the puborectalis for this novel approach. For those tumors of which the lowest border is less than $1 \mathrm{~cm}$ from the puborectalis noted on preoperative MRI or intraoperatively, abdominoperineal resection is usually offered as we do not routinely perform intersphincteric resection. We also have a multidisciplinary team comprising of surgeons, medical oncologists, radiation oncologists,

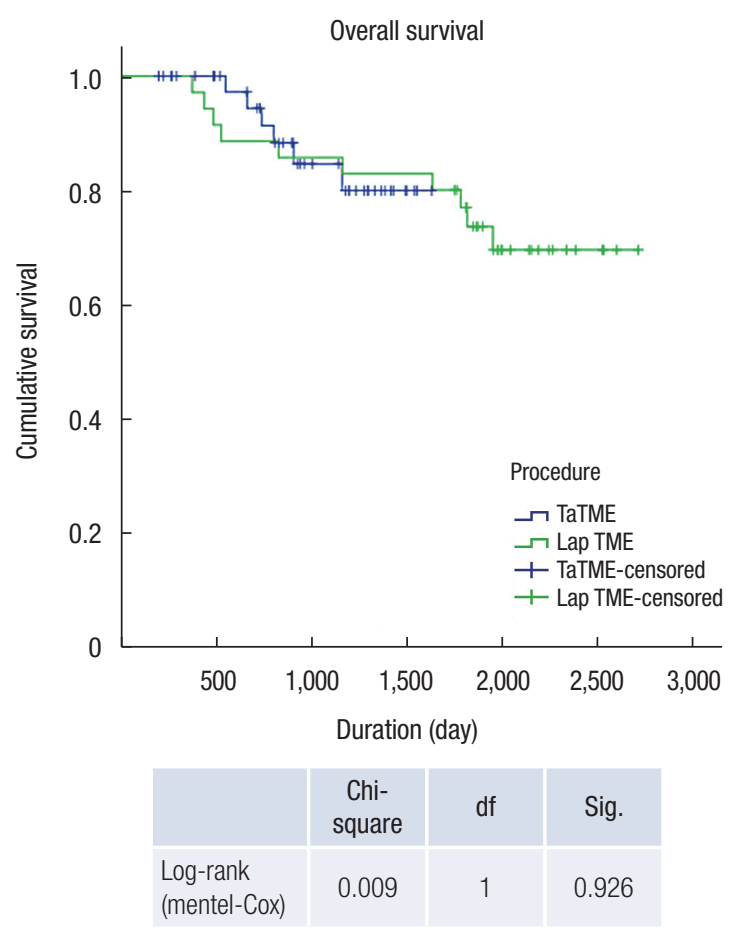

Fig. 6. Kaplan-Meier curve of overall survival of laparoscopic (Lap) total mesorectal excision (TME) vs. transanal TME (TaTME). df, degree of freedom; Sig., significance.

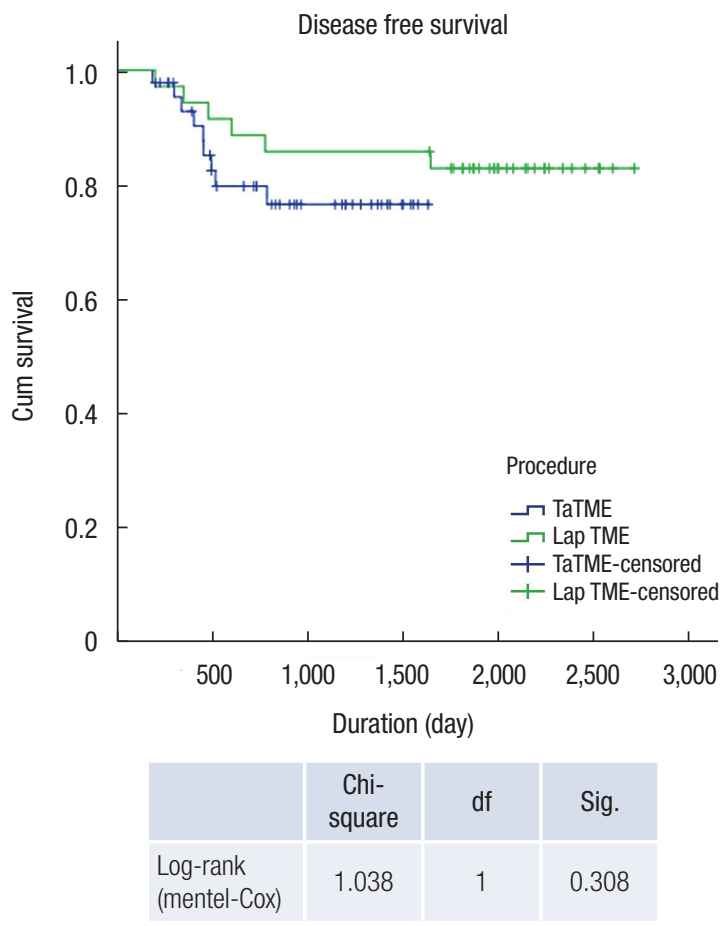

Fig. 7. Kaplan-Meier curve of disease-free survival of laparoscopic (Lap) total mesorectal excision (TME) vs. transanal TME (TaTME). df, degree of freedom; Sig., significance. 
radiologists, and pathologists to determine whether neoadjuvant treatment is needed before we operate on patients. Patients with resectable solid organ metastasis are regarded as having resectable disease and radical resection will be offered as they have a better 5-year or even 10-year survival [14].

In our unit, about $80 \%$ of all colorectal cancer resections are performed using the minimally invasive or laparoscopic approach, and our average conversion rate is $8 \%$. However, in the present study involving only low rectal cancer patients, the conversion rate of the Lap TME group was up to $20 \%$. Five out of 7 conversions $(71.4 \%)$ were due to difficult pelvic conditions including bleeding in the pelvis, visceral obesity, and difficulty in rectal transection. These are well recognized technical limitations of Lap TME and are very challenging to deal with $[2,15,16]$. The fact that more than 3 stapler firings were required to transect the lower rectum in the Lap TME group in our study has illustrated the technical difficulty associated with rectal surgery in the deep pelvis. According to published data from international trials, the conversion rate of laparoscopic rectal resection ranges from $2 \%$ to $38 \%[2,17,18]$. Our conversion rate is probably on the high side of the published series. However, the patients whom we selected to compare against TaTME were those with low rectal tumors $(\leq 5$ $\mathrm{cm}$ from the puborectalis) who underwent Lap TME-this is the group that is technically most difficult to tackle in terms of dissection and transection, and therefore their conversion rate is expected to be higher. With the introduction of TaTME, these technical difficulties can be potentially overcome, and remarkably our study has shown a low conversion rate of $4.4 \%$ associated with TaTME. This observation has also been demonstrated in different published series of TaTME for low rectal cancer, and even expert's opinion in the St. Gallen Consensus statement 2017 has fully acknowledged this issue $[5,18,19]$. In this regard, the implementation of TaTME can be highly beneficial not only to expert centers but also to medium volume colorectal units to overcome technical difficulties associated with conventional Lap TME.

In our study, the mean operating time of the TaTME group was 30 minutes longer than that of the Lap TME group. The operating times of the 2 procedures plotted against the number of cases were shown in Fig. 5. There can be a few reasons to explain this observation. First, we are probably still on our learning curve. The transanal surgeon has to face different anatomy and he/she has to undergo adequate training in order to avoid the complications related to this new technique. Second, we are a medium volume unit with only one consultant colorectal surgeon who is fully trained in TaTME. We are not using a 2-team simultaneous approach for most of our cases. We routinely commence our operation with the abdominal team dissection first. The transanal team can only start after the splenic flexure has been completely mobilized. Then the rectal dissection will be performed transanally with some abdominal assistance. This can potentially lengthen the operating time. However, the operating time of the TaTME group is approaching that of the conventional Lap TME toward the end of the series, indicating a learning curve effect (Fig. 5). The operating time can be further reduced if the procedure can be performed using a two-team simultaneous approach. Unfortunately, for a medium volume colorectal unit like ours with limited number of specialist surgeons available, a 2-team approach is not always possible. Third, there is also a learning curve for the whole operating theater team (including the scrub nurses and the circulating staff) to be familiar with the procedure and different operative steps in order to achieve a smooth operation with faster operating time. TaTME is a team operation and everyone in the theater is a vital part of the team for a successful and smooth operation.

The mean number of stapler firings used to transect the lower rectum in the Lap TME group was more than 3, which is not favorable. This reflects the technical difficulty in distal transection of low rectal tumor located within $5 \mathrm{~cm}$ from the puborectalis. It would be even more difficult in the presence of other unfavorable factors such as narrow pelvis, obese patients, and large pelvic tumor. This is one of the reasons why we adopted TaTME to tackle the problem of difficult transection in these circumstances. Our results showed that TaTME can reduce the risk of conversion to open laparotomy for transection, and possibly achieve a lower resection margin positivity rate when compared with Lap TME.

The mean tumor location was significantly lower in the TaTME group than in the conventional Lap TME group on preoperative MRI images. However, the mean distal margin was not compromised in the resected TaTME specimens. This indicated that we have pushed the limited of sphincter preservation further to include more distally locating tumors. The implementation of TaTME a medium volume unit could potentially improve sphincter preservation without compromising the quality of surgical resection. This avoids conversion to abdominoperineal resection in cases of which distal rectal transection with adequate margin is not possible with the conventional top-down approach. Metaanalysis on pathologic outcomes suggested that TaTME does provide better distal margins and circumferential resection margins when compared with conventional Lap TME $[20,21]$. By direct visualization of the distal end of the tumor, the distal resection margin can be ascertained before the rectum is transected. There is also no difference in the overall margin positivity rate.

TaTME is a novel surgical approach, and novel complications associated with it have been reported in the literature since its introduction $[4,5,19]$. These include urethral injury, vascular injury in the lateral compartment, and abnormal pattern of local recurrence. In our study, there was no difference in major complications such as anastomotic leak and reoperation between the 2 groups, but there were higher incidence rates of prolonged ileus and UTI/AROU in TaTME group. There was no urethral or vaginal injury. We also did not record an abnormal pattern of recurrence as documented in the Norwegian series [4].

TaTME provides colorectal surgeons with an additional armamentarium to deal with difficult rectal cancer cases. Large registry 
data proved that the technique is oncologically safe and effective [22]. The international consensus statement [19] suggested that proper training should consist of hands-on courses, proctoring program, and proper supervision in the first 1 to 5 cases. The learning curve is estimated to be around 20 cases. With proper training, the benefits of this technique can be delivered to both colorectal surgeons and patients at medium volume colorectal units like ours. After initial training, the mean operating time of our initial series of TaTME was only 30 minutes more than that of conventional Lap TME, a technique of which we have been doing for many years. We are probably still on the learning curve in terms of operating time. However, our study has clearly demonstrated that TaTME can benefit both colorectal surgeons and patients by offering easier distal rectal transection, lower conversion rate, possibly lower resection margin positivity, and similar clinical outcomes when compared with Lap TME.

The main limitation of this study is its retrospective nature. Ideally, the 2 approaches should be evaluated by a prospective randomized trial. However, as a medium volume center, patient recruitment for a prospective trial is anticipated to be slow and difficult. Nevertheless, the current study has demonstrated that it is safe to adopt this new approach after proper training, with proven benefits for patients including lower conversion rate and possibly lower resection margin positivity.

In conclusion, it is technically feasible and oncologically safe to perform TaTME in a medium volume colorectal unit after adequate training. This new approach can benefit patients with difficult pelvic anatomy, reduce the risk of conversion, potentially reduce margin positivity rate, and push the sphincter preservation limits for low rectal cancer.

\section{CONFLICT OF INTEREST}

No potential conflict of interest relevant to this article was reported.

\section{FUNDING}

None.

\section{RERERENCES}

1. Sylla P, Rattner DW, Delgado S, Lacy AM. NOTES transanal rectal cancer resection using transanal endoscopic microsurgery and laparoscopic assistance. Surg Endosc 2010;24:1205-10.

2. Targarona EM, Balague C, Pernas JC, Martinez C, Berindoague R, Gich I, et al. Can we predict immediate outcome after laparoscopic rectal surgery? Multivariate analysis of clinical, anatomic, and pathologic features after 3-dimensional reconstruction of the pelvic anatomy. Ann Surg 2008;247:642-9.

3. Chang TC, Kiu KT. Transanal total mesorectal excision in lower rectal cancer: comparison of short-term outcomes with conven- tional laparoscopic total mesorectal excision. J Laparoendosc Adv Surg Tech A 2018;28:365-9.

4. Wasmuth HH, Faerden AE, Myklebust TÅ, Pfeffer F, Norderval S, Riis R, et al. Transanal total mesorectal excision for rectal cancer has been suspended in Norway. Br J Surg 2020;107:121-30.

5. Hasegawa H, Okabayashi K, Tsuruta M, Ishida T, Asahara F, Coleman MG. Evolution of surgery for rectal cancer: Transanal total mesorectal excision new standard or fad? . J Anus Rectum Colon 2018;2: 115-21.

6. Deijen CL, Velthuis S, Tsai A, Mavroveli S, de Lange-de Klerk ES, Sietses C, et al. COLOR III: a multicentre randomised clinical trial comparing transanal TME versus laparoscopic TME for mid and low rectal cancer. Surg Endosc 2016;30:3210-5.

7. Lelong B, de Chaisemartin C, Meillat H, Cournier S, Boher JM, Genre D, et al. A multicentre randomised controlled trial to evaluate the efficacy, morbidity and functional outcome of endoscopic transanal proctectomy versus laparoscopic proctectomy for low-lying rectal cancer (ETAP-GRECCAR 11 TRIAL): rationale and design. BMC Cancer 2017;17:253.

8. Marks JH, Nassif GJ, Marks G. Transanal abdominal transanal proctosigmoidectomy with descending coloanal anastomosis (the TATA procedure) for low rectal cancer treated with chemoradiation. In: Schiessel R, Metzger P, editors. Intersphincteric resection for low rectal tumors. Wien: Springer; 2012. p. 153-8.

9. Serra-Aracil X, Mora-López L, Casalots A, Pericay C, Guerrero R, Navarro-Soto S. Hybrid NOTES: TEO for transanal total mesorectal excision: intracorporeal resection and anastomosis. Surg Endosc 2016;30:346-54.

10. Atallah $\mathrm{S}$. Transanal minimally invasive surgery for total mesorectal excision. Minim Invasive Ther Allied Technol 2014;23:10-6.

11. van Gijn W, Gooiker GA, Wouters MW, Post PN, Tollenaar RA, van de Velde CJ. Volume and outcome in colorectal cancer surgery. Eur J Surg Oncol 2010;36 Suppl 1:S55-63.

12. Hospital Authority. Surgical Outcomes Monitoring \& Improvement Program (SOMIP) report. Vol. 11. Hong Kong: Hong Kong SAR Government; 2019.

13. Deijen CL, Tsai A, Koedam TW, Veltcamp Helbach M, Sietses C, Lacy AM, et al. Clinical outcomes and case volume effect of transanal total mesorectal excision for rectal cancer: a systematic review. Tech Coloproctol 2016;20:811-24.

14. Creasy JM, Sadot E, Koerkamp BG, Chou JF, Gonen M, Kemeny NE, et al. Actual 10-year survival after hepatic resection of colorectal liver metastases: what factors preclude cure? Surgery 2018;163:1238-44.

15. Cecil TD, Taffinder N, Gudgeon AM. A personal view on laparoscopic rectal cancer surgery. Colorectal Dis 2006;8 Suppl 3:30-2.

16. Emile SH, de Lacy FB, Keller DS, Martin-Perez B, Alrawi S, Lacy AM, et al. Evolution of transanal total mesorectal excision for rectal cancer: from top to bottom. World J Gastrointest Surg 2018;10: 28-39.

17. Kang SB, Park JW, Jeong SY, Nam BH, Choi HS, Kim DW, et al. Open versus laparoscopic surgery for mid or low rectal cancer af- 
ter neoadjuvant chemoradiotherapy (COREAN trial): short-term outcomes of an open-label randomised controlled trial. Lancet Oncol 2010;11:637-45.

18. van der Pas MH, Haglind E, Cuesta MA, Fürst A, Lacy AM, Hop WC, et al. Laparoscopic versus open surgery for rectal cancer (COLOR II): short-term outcomes of a randomised, phase 3 trial. Lancet Oncol 2013;14:210-8.

19. Adamina M, Buchs NC, Penna M, Hompes R; St. Gallen Colorectal Consensus Expert Group. St. Gallen consensus on safe implementation of transanal total mesorectal excision. Surg Endosc 2018;32:1091-103.

20. Jiang HP, Li YS, Wang B, Wang C, Liu F, Shen ZL, et al. Pathologi- cal outcomes of transanal versus laparoscopic total mesorectal excision for rectal cancer: a systematic review with meta-analysis. Surg Endosc 2018;32:2632-42.

21. Roodbeen SX, Penna M, Mackenzie H, Kusters M, Slater A, Jones OM, et al. Transanal total mesorectal excision (TaTME) versus laparoscopic TME for MRI-defined low rectal cancer: a propensity score-matched analysis of oncological outcomes. Surg Endosc 2019;33:2459-67.

22. Penna M, Hompes R, Arnold S, Wynn G, Austin R, Warusavitarne J, et al. Transanal total mesorectal excision: international registry results of the first 720 cases. Ann Surg 2017;266:111-7. 